\title{
Exploration and Practice for Fusion Cultivation Mode of Innovative Talents Majoring in Biomedical Engineering
}

\author{
Peng Anmin, Hu Zhaoyan, Lyu Jie \\ College of Medical Instruments \\ Shanghai University of Medicine \& Health Sciences \\ Shanghai, China \\ 106410923@qq.com
}

\begin{abstract}
Based on current situations in biomedical engineering discipline and existing problems of cultivation mode for talents majoring in biomedical engineering, the paper, combing new-rising discipline characteristics of multidisciplinary integration for biomedical engineering and cultivation requirements of Innovative Biomedical Engineers, by virtue of innovative talents cultivation mode and experience of domestic and overseas universities, and adhering to the cultivation concept of fusion innovation, explores and practices respectively from cultural environment, general and major fusion, scientific research and teaching fusion, medicine and engineering fusion as well as internal and external fusion. So the paper frequently forms a fusion cultivation mode of innovative patents majoring in biomedical engineering keeping up with the times.
\end{abstract}

Keywords-Biomedical engineering; Innovative talents; Fusion cultivation mode

\section{INTRODUCTION}

Biomedical engineering is a new-rising discipline of natural science, medicine and engineering by cross fusion, including many major orientations. The universities set with biomedical engineering major both at home and abroad generally set course systems and teaching content of the major based on original resources of the university and the course systems and teaching contents of biomedical engineering major for engineering, science and medicine universities have different focuses, even though the courses of same universities, the settings are quite different [1]. Course system of biomedical engineering major for mangy universities is simple listing of science, engineering and medicine courses, which is short of both classical general course and the course fusing science, engineering and medicine and has problems such as continuous use of the management system and evaluation mode of common discipline in management system and evaluation mode and disjointed theory and practice, separated science research and teaching and no complementary campus teaching and off-campus teaching etc. in cultivation mode and teaching method and means [2-4]. In addition, the universities have ambiguous location against that what kind of talents majoring in biomedical engineering are to be cultivated and haven't form the talents cultivation mode suitable for the major yet. Due to cross fusion of biomedical engineering discipline, the talents cultivated for the discipline are inter-disciplinary talents certainly; what's more, since biomedical engineering discipline is a new-rising one and has strong innovation, rapid development speed and fast application of new materials and new technologies, the talents cultivated for the discipline should be innovative talents. Therefore, the talents cultivation purpose of the major should be inter-disciplinary innovative talents. According to the characteristics of biomedical engineering discipline and talents cultivation problems above, Shanghai University of Medicine \& Health Sciences adheres to fusion and innovation concepts in cultivation of talents majoring in biomedical engineering, focuses on the cultivation specification of innovative applied talents, actively uses advanced cultivation modes and experience of domestic and overseas universities for reference and is forming a fusion cultivation mode of innovative talents majoring in biomedical engineering frequently with its own characteristics.

\section{FORMING OF FUSION CULTIVATION MODE FOR INNOVATIVE TALENTS MAJORING IN BIOMEDICAL ENGINEERING}

\section{A. Actively create a cultural environment to cultivate innovation thoughts and spirits}

Famous "Qian Xuesen's Question" - "Why do our universities always fail to produce excellent talent?" pointedly questions the environmental subject to cultivate talents university, which has no democratic atmosphere and inappropriate cultivation mode. The solution of "Qian Xuesen's Question" also points out directly that improvement should be made in national policy, cultivation mode, democratic atmosphere etc [5]. Substantially, the solution of "Qian Xuesen's Question" is to create a cultural environment breeding innovation thought and spirit firstly. Creation of the cultural environment breeding innovation thought and spirit requires joint effect of the nation, society and university. In university, it is mainly to create a strong academic innovation atmosphere of democracy and freedom between students and teachers. Massachusetts Institute of Technology (MIT) established "Undergraduate Research Opportunities Program 
(UROP)" in 1969 and developed "Independent Activity Program (IAP)" later, which is an intelligence collaboration program of the undergraduates and teachers and in which the undergraduates can choose to participate in teacher's research subject or design research subject themselves and invite the teachers as a Counselors according to their own interests. IAP provides an open opportunity for undergraduates to participate in research as the colleagues of teacher. To effectively implement the program, the university provides 1-month independent time for teachers and students to put themselves in the topic research with USD 5,000,000 provided as the salaries of students participating in undergraduate research program [67]. These measures of MIT obtain significant effect and many universities follow soon because implementation of these programs creates an academic innovation atmosphere and innovation culture environment with democracy and freedom. In addition, many universities support different levels and kinds of innovation competitions and form an upsurge driving innovation by competitions in the campus. Other measures to create a cultural environment breeding innovation thought and spirit include: open laboratory to teachers and students, build a laboratory platform with originality \& innovation, perfect information network and book digital resource of universities, add public courses, online courses and online learning platform of various disciplines and customize mobile learning APP to forge a seamless campus mobile leaning platform, hold all kinds of seminars, academic salons, academic forums, academic reports etc. In recent years, biomedical engineering major of Shanghai University of Medicine \& Health Sciences actively organizes students to participate in various competitions, such as 3D Design Show, China "Internet Plus" Innovation and Entrepreneurship Competition for College Students, "Challenge Cup" National College Student Competition, Shanghai Electronic Product Design and Production of National Vocational Student Skills, Shanghai College Student Hack Competition, "Shangtu Cup" Advanced Picture Drawing Competition etc. and obtains excellent marks.

\section{B. Promote the fusion of general education and major education to improve humanistic and artistic cultures of students}

In university education, university should provide sufficient resources and helps for students for development of individual specialties and interests \& hobbies. Even though individual specialties and interests \& hobbies are different from the major leaned, paying attention to personality cultivation of students is of great help to innovation of major engaged by the students [8]. Science and technology serve for human and only when the talents fond of humanistic and social science and majoring in science, engineering and medicine add humanity and art elements or factors to major innovation, can they lead the innovation.

For cultivation of innovative talents majoring in biomedical engineering, the fusion of major education and general education as well as humanistic and artistic cultures of biomedical engineering may be strengthened from the following three aspects: firstly, add compulsory general course in course system and provide many humanistic and artistic courses at same time for optional courses of students; secondly, open corresponding resources for and provide support to students with humanistic and artistic specialties and hobbies \& interests, such as encouraging students complying with conditions to enter in second major leaning to conduct targeted individualized training etc.; thirdly, encourage and support communication between students majoring in biomedical engineering and students majoring in humanity and art and teachers, such as encouraging and supporting them to participate in humanistic and artistic associations, training class etc. and create various convenient conditions for communications between teachers and students.

\section{Promote the fusion of scientific research and teaching to improve students' innovation quality}

Primary task of university is to cultivate talents with innovation quality and teaching and scientific research should be two means for cultivation of talents. Many universities assess the teachers mainly from scientific research and teaching performances, with the trend of treasuring scientific research and despising teaching generally and no good fusion of scientific research and talents cultivation during talents cultivation [2].

To change the adverse conditions above: firstly, in system, change actually existing evaluation system and policy of university on treasuring scientific research and despising teaching, break the separation of teaching and scientific research, integrate teaching resources and scientific research resources and mobilize the initiative of students and teachers to cultivate innovative composite talents; encourage teachers to translate advanced discipline knowledge and engaged scientific research achievements into effective teaching contents, implement UROP or IAP, guide the students to participate in competitions etc. so as to achieve the purposes to fuse teachers and students, teaching and scientific research and cultivate innovative talents. Shanghai University of Medicine \& Health Sciences strongly supports innovative talents cultivation of biomedical engineering major, encourages cooperation and communications among various colleges and affiliated hospitals and improves students' innovative quality and cultivates innovative talents by integrating related resources, fusing scientific research and teaching and forming complementary advantages.

\section{Promote the fusion of medicine and engineering to cultivate Innovative Biomedical Engineers}

Primary task of biomedical engineering major is to cultivate Biomedical Engineers. Career goal of Biomedical Engineer is to know, transform or control biological system with electricity, chemistry, optics, mechanics and other engineering discipline principles, knowledges and methods to solve special questions related to medical treatment. Therefore, Biomedical Engineer should be provided with sufficient biomedicine knowledges and master important basic engineering technology at same time. Biomedical Engineer should integrate into medical treatment practice and closely cooperate with Medical Workers and Biological Scientist and their career activity is the practice of medical and engineering fusion [9]. To cultivate Innovative Biomedical Engineers, the universities must walk the cultivation route fusing medicine and engineering. The 
universities may prepare the teaching plan (cultivation scheme) of biomedical engineering major according to their own feature location, but the teaching plan must reflect the cultivation route fusing medicine and engineering. The biomedical engineering major of Shanghai University of Medicine \& Health Sciences is established based on medical instruments engineering majors, with the tradition fusing medicine and engineering. To have its own features, biomedical engineering major team makes a lot of researches, including careful analysis, comparison and research the cultivation schemes of universities for biomedical engineering major and sufficient research of industrial demands and prepares the cultivation scheme of biomedical engineering major deeply fusing medicine and engineering by combing the characteristics of biomedical engineering discipline, such as cross, innovation, strong practice and rich medicine and engineering resources of the university and conforming to trend of the times and social demands, which not only reflects establishment of biomedical engineering personnel's values such as moral principles etc., but also pays attention to acquisition of basic knowledge and skills of biomedical engineering major.

\section{E. Promote the fusion of the internal and the external to cultivate Innovative Biomedical Engineers}

Characteristics of biomedical engineering decide on that cultivation of innovative talents of the major should strengthen major and non-major resources fusion, deep campus and offcampus cooperation and deep domestic and overseas cooperation to form an internal and external fusion cultivation mode. The universities shall encourage establishment of a teaching and scientific research team and scientific research and teaching platform crossing biomedical engineering major and integrate various resources to cultivate Innovative Biomedical Engineers by fusion; actively carry out deep cooperation between the university and enterprises, hospitals, other universities, scientific research institutions, industrial supervision departments etc. to cultivate Innovative Biomedical Engineers by fusion. Main deep cooperation forms of university and enterprise (hospital) include joint laboratory, off-campus training base, technology research and development etc. "Blood Purification Training Center of Shanghai University of Medicine \& Health Sciences" established by Shanghai University of Medicine \& Health Sciences, Chinese Non-government Medical Institutions Association and Shangyao Yisheng Medical Investment and Management (Shanghai) Co., Ltd. by cooperation and "Operation and First Aid Engineering Technical Laboratory" jointly established by Shanghai University of Medicine \& Health Sciences and Stryker (China) Corporation are typical internal and external fusion cultivation bases of Biomedical Engineers. Established in College of Medical Instruments of Shanghai University of Medicine \& Health Sciences, the two bases integrate high-quality resources in and off of the campus. "Blood Purification Training Center of Shanghai University of Medicine \& Health Sciences" employs well-known experts as central teachers to bear actual training task of students and carry out external training. Configured with four teaching zones, i.e. pre-hospital first aid, in-hospital transfer, ICU and delivery room and conventional operating room \& integrated operating room and integrating high and new product technology of Stryker and advanced teaching concepts of universities, "Operation and First Aid Engineering Technical Laboratory" is not only a medicine and engineering fusion platform but also an internal and external fusion platform, which promote fusion cultivation of Innovative Biomedical Engineers. Main deep cooperation forms between universities (institutes) include academic cooperation, course introduction, cooperative cultivation etc., such as deep cooperation between the university and Furtwangen University of Applied Sciences in surgical technique of heart, cardiopulmonary bypass technique etc. and Osaka University etc. in medicine and engineering fusion fields. In addition, the university also actively implements internal and external fusion cultivation of Innovative Biomedical Engineer projects such as overseas research project of teachers and students for biomedical engineering major. Through internal and external fusion cultivation, the practical application capacity of students majoring in biomedical engineering is improved significantly, innovative thinking is trained systematically and international view is widened greatly.

\section{CONCLUSION}

Fusion cultivation mode of innovative talents majoring in biomedical engineering has distinctive characteristics and significant effect. Along with technical progress and social development, the Teachers Team of biomedical engineering major should update cultivation conception, track discipline front, learn advanced experience, integrate high-quality resources, dare to exploration and practice and continuously improve fusion cultivation mode of innovative talents majoring in biomedical engineering so that it is improved gradually.

\section{REFERENCES}

[1] Cai Zhanxiu, Qiu Zhaoyun, Gao Xianghong and Zhao Renhong, Exploration and Research on Cultivation Mode of Undergraduates Majoring in Biomedical Engineering [J], China Education Technology and Equipment, 2016 (20):87-89. (In Chinese)

[2] Chen Hongbo, Applied Talents Cultivation Mode Fusing Scientific Research and Teaching for Biomedical Engineering Major - Exampled by Guilin University of Electronic Technology [J], Journal of Higher Education, 2016(13):63-64. (In Chinese)

[3] Chen Hongbo, Talents Cultivation System and Practice of Enterprises and Hospitals for Fusion in Biomedical Engineering Major [J], Education and Teaching Forum, 2016(42):1-3. (In Chinese)

[4] Wu Dexing, Cultivation of Innovative Talents by Fusion of Scientific Research and Teaching [J], China University Teaching, 2014(1):4-7. (In Chinese)

[5] Xu Yuehong and Yue Xianping, the Connotation, Essence and Effect of "Qian Xuesen's Question" - Discussion with Professor Lin Yanzhi [J], Journal of the Chinese Society of Education, 2014(12):1-5,21. (In Chinese)

[6] He Mi, Li Yongqin, Chen Bihua and Li Yi, Research on Scientific Research Capacity Cultivation of Undergraduates Majoring in Biomedical Engineering $[\mathrm{J}]$, Northwest Medical Education, 2014(4):660-663. (In Chinese)

[7] You Fusheng, Xu Canhua, Yang Bin, Li Yan, Tang Chi, Ma Xiaoyu, Liu Jing and Li Wenbin, Educational Concept of Massachusetts Institute of Technology and the Enlightenment to Biomedical Engineering Major [J] Chinese Medical Equipment Journal, 2016(1):144-146. (In Chinese)

[8] Xu Huiwen, Reformation and Exploration on Cultivation of Innovative Talents of Universities from Fusion of Science and Art [J], Chinese University Technology Transfer, 2015(4):94-96. (In Chinese)

[9] (The United States) Written by John D. Enderle and Joseph D. Bronzino, translated by Feng Zhouyan, Introduction to Biomedical Engineering (Original 3rd Edition) [M], Beijing: China Machine Press, 2014:17-18. (In Chinese) 